\title{
Coronavirus disease 2019 (covid-19): a guide for UK GPs
}

\author{
Mohammad S Razai academic clinical fellow in primary care ${ }^{1}$, Katja Doerholt consultant in paediatric \\ infectious diseases ${ }^{2}$, Shamez Ladhani consultant in paediatric infectious diseases and public health ${ }^{3}$, \\ Pippa Oakeshott professor of general practice ${ }^{1}$
}

${ }^{1}$ Population Health Research Institute, St George University of London, London, UK; ${ }^{2}$ St George's University Hospital NHS Foundation Trust, London, UK; ${ }^{3}$ Public Health England, London, UK

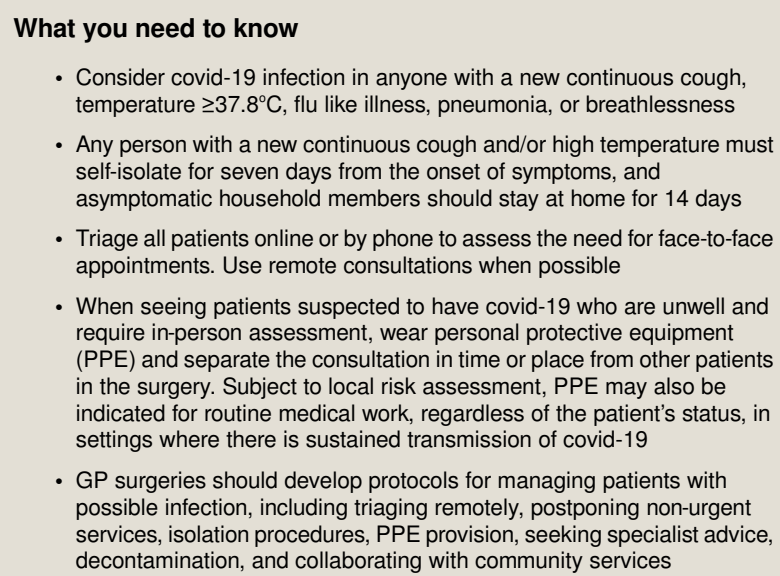

- GP surgeries should develop protocols for managing patients with possible infection, including triaging remotely, postponing non-urgent services, isolation procedures, PPE provision, seeking specialist advice, decontamination, and collaborating with community services

The UK recorded its first confirmed case of acute respiratory infection due to coronavirus disease 2019 (covid-19) on 31 January 2020 and initially responded by quarantining at-risk individuals to contain the spread of infection. However, when covid-19 cases increased and containment was no longer effective, the UK moved to a delay phase of the response on 12 March 2020. Executive agencies Public Health England (PHE), ${ }^{1}$ Health Protection Scotland (HPS), and NHS England have since published updated guidance for healthcare providers on managing patients suspected to have covid-19. Guidance for the public and health professionals varies internationally, depending partly on risk levels and healthcare systems, and is being regularly updated.

This article offers a practical guide for GPs and others working in UK primary care on when to suspect covid-19 and how to respond. It is based on current UK guidance at the time of publication and was updated on 3 April 2020. We recommend readers consult the latest guidance (box 1).

\section{Box 1: Essential resources}

Covid-19: latest case definition, investigation, and initial clinical management of possible cases:

https://www.gov.uk/government/publications/wuhan-novel-coronavirusinitial-investigation-of-possible-cases/investigation-and-initial-clinicalmanagement-of-possible-cases-of-wuhan-novel-coronavirus-wn-covinfection

Coronavirus: latest information and advice:

https://www.gov.uk/guidance/wuhan-novel-coronavirus-information-forthe-public

Guidance for households with possible coronavirus infection:

https://www.gov.uk/government/publications/covid-19-stay-at-homeguidance/stay-at-home-guidance-for-people-with-confirmed-or-possiblecoronavirus-covid-19-infection

Guidance on social distancing and for vulnerable people:

https://www.gov.uk/government/publications/covid-19-guidance-on-socialdistancing-and-for-vulnerable-people

Guidance on infection prevention and control for covid-19:

https://www.gov.uk/government/publications/wuhan-novel-coronavirusinfection-prevention-and-control

Covid-19: interim guidance for primary care (including environmental cleaning after possible case):

https://www.gov.uk/government/publications/wn-cov-guidance-for-primarycare/wn-cov-interim-guidance-for-primary-care

Covid-19: latest guidance for primary care on Health Protection Scotland (HPS):

https://www.hps.scot.nhs.uk/a-to-z-of-topics/covid-19/

World Health Organization (WHO): technical documents for coronavirus (covid-19) outbreak:

https://www.who.int/health-topics/coronavirus

European Centre for Disease Prevention and Control: latest guidance for EU/EEA: https://www.ecdc.europa.eu/en/novel-coronavirus-china

US Centers for Disease Control and Prevention: latest guidance, advice and information: https://www.cdc.gov/coronavirus/2019-ncov/index.html 


\section{What do we know about the clinical course of covid-19?}

The median estimated incubation period is five to six days (range 0 to 14 days). ${ }^{2}$ The median age of patients with a confirmed case is around 59 years. ${ }^{3}$ In adults, the most common symptoms at presentation are cough (68\%), fever (44\%), fatigue (38\%), myalgia/arthralgia (15\%), and headache (14\%). ${ }^{4}$

Initial data indicate that more than $80 \%$ of patients have asymptomatic to moderate disease and recover, but about $15 \%$ may get severe disease including pneumonia, and around 5\% become critically unwell with septic shock and/or multi-organ and respiratory failure. ${ }^{56}$ The case fatality rate is estimated at approximately $2 \%$ overall, but ranges from $0.2 \%$ in people under 50 to $14.8 \%$ in those over 80 , and is higher among those with chronic comorbid conditions. ${ }^{6}$

\section{When to consider covid-19}

Consider covid-19 in anyone requiring hospital admission with any of the following:

- flu like illness: fever $\geq 37.8^{\circ} \mathrm{C}$ and at least one of the following respiratory symptoms of acute onset: - persistent cough (with or without sputum)

- hoarseness, nasal discharge or congestion, shortness of breath, sore throat, wheezing, sneezing

- acute respiratory distress syndrome

- either clinical or radiological evidence of pneumonia. ${ }^{7}$

Clinicians should be alert to the possibility of atypical presentations in patients who are immunocompromised.

Otherwise, consider covid-19 in anyone who has either a new continuous cough or a high temperature, or both, but be aware that the infection can present with mild and non-specific viral symptoms.

\section{What are the public being asked to do if they think they are infected?}

In the UK, the public are advised not to attend GP surgeries, community pharmacies, or hospitals if they have a new continuous cough, temperature $\geq 37.8^{\circ} \mathrm{C}$, or flu like illness. Instead, they are being advised to self-isolate at home for seven days from onset of their symptoms and only contact NHS 111, the national non-emergency helpline, if symptoms worsen during home isolation or if they are not better after seven days. Asymptomatic members of the household should stay at home for 14 days starting from the day when the first person in the house became ill.

GP surgeries should put measures in place to reinforce this approach by

- triaging all patients online or by phone (box 2)

- displaying large posters at the entrance to GP surgeries and a prominent notice on websites and online booking systems

- using pre-recorded messages on telephone systems

- sending patients SMS (text) messages.
Box 2: How to respond if you suspect covid-19 exposure or infection during a telephone consultation

If a patient calls for advice from home or elsewhere and covid-19 is suspected: - Assess whether they are clinically stable and not critically unwell. If the patient is critically unwell and requires urgent transfer to hospital, call 999 and inform the ambulance call handler of a suspected case of covid-19

- Avoid face-to-face assessment in primary care including out-of-hours centres and GP hubs. Advise the patient to self-isolate for seven days and give "Stay at Home" advice (see resources, box 1). Advise them to contact NHS 111 only if symptoms get worse

- If the patient is severely unwell and hospital care is being considered, follow your local pathway for referral to secondary care for possible assessment in hospital

- If hospital assessment is advised, agree a method of transport with the hospital team, such as the patient's own car or an ambulance. Patients should not use public transport or taxis to get to hospital

- Further guidance on transport to hospital is available on PHE/HPS website (see resources, box 1)

If patients arrive at reception without prior arrangement they should be asked if they or anyone in their household has a high temperature or a new continuous cough. Those who answer "yes" should be advised to go home, self-isolate, and follow NHS coronavirus advice. ${ }^{7}$ If they are unable to cope at home and therefore unable to follow this advice, they should be immediately isolated at the practice (box 3) and considered for possible referral to secondary care.

Box 3: How to isolate acutely unwell patients with suspected covid-19 in primary care

- Isolate individuals suspected to have covid-19, their waste, and their belongings in a room with the door closed and window open (switch off any air conditioning)

- The patient should wear a fluid resistant surgical mask (Type IIR), if tolerated, during transportation, in clinical areas, and in communal waiting areas. A mask should not be used if clinical care will be compromised, such as when receiving oxygen therapy via a mask

- Plan in advance which room is most appropriate for isolation and care. It should ideally be located away from the waiting area and other consultation rooms. Avoid a room with carpeted floors or soft furnishing as these are difficult to decontaminate

- The room should be clutter free with equipment kept in closed cupboards. Tie back examination curtains and arrange regula laundering

- If contact with a patient for assessment or treatment is necessary, wear standard PPE (box 4)

- The patient must not use the surgery's communal toilets. A toilet facility should be reserved, preferably close to the isolation room. Advise the patient not to touch anything or anyone when walking to the toilet and to wash hands thoroughly afterwards

- Communicate with the isolated patient preferably by phone or a conversation through the closed door to reassure them and provide updates. Follow your local pathway for seeking advice and referral

\section{Patients who need face-to-face assessment}

Patients who have been assessed remotely, are suspected to have covid-19, and who need face-to-face assessment in the surgery should be segregated in place or time from other patients through separate entrances, designated areas in the practice, or seen at a different time (for example, at the end of the list). Where this is not practical, follow local protocols for further assessment and possible hospital admission. Staff must wear standard PPE during assessment of this cohort of patients and follow infection prevention and control guidance.

If a home visit is unavoidable, wear PPE for face-to-face assessment and consult infection prevention and control guidance (see resources, box 1), as additional precautions may be needed for some patients, such as those on non-invasive 
ventilation and those requiring aerosol generating procedures. ${ }^{7}$ If you identify two or more possible or confirmed cases of covid-19 from a long-term care facility, inform the local Health Protection Team. ${ }^{78}$ Extremely vulnerable groups such as solid organ transplant recipients, individuals on immunosuppression therapies, and those with severe asthma and COPD must take shielding measures. These include not leaving their home for 12 weeks from the day they are notified by NHS England, using remote consultations where possible, and strictly avoiding contact with people displaying covid-19 symptoms. ${ }^{9}$ Health professionals should wear apron, gloves, and a surgical mask (which does not need to be fluid resistant type IIR) when they see individuals (or someone in their household) who are in the extremely vulnerable group undergoing shielding. ${ }^{10}$

\section{What if the patient is severely unwell?}

If the patient is critically ill, call 999 and advise the call handler that the patient may have covid-19 infection. ${ }^{11}$ If you make a clinical judgment that the patient needs further assessment or treatment while awaiting transfer to hospital, bear in mind your own safety and those around you and wear PPE as described in box 4.

\footnotetext{
Box 4: Personal protective equipment for covid-19 in primary care $^{10}$

- For any direct care (within two metres) of people with possible or confirmed infection, or for collection of nasopharyngeal swab(s), wear protective clothing in line with the standard infection control precautions (gloves, apron, a fluid resistant surgical mask (type IIR), and goggles or visor) and keep exposure to a minimum

- Subject to local risk assessment, PPE may also be indicated for work in settings where there is sustained transmission of covid-19

- Aprons and gloves are for single use only. A fluid resistant surgical mask and eye protection can be for single use or used for a full session. A session ends when the healthcare worker leaves the clinical care setting

- All protective clothing and equipment should be disposed of as clinical waste
}

\section{After the patient leaves the surgery}

Follow detailed guidance (box 1) to ensure that the room is cleaned correctly. The person(s) cleaning the room should wear a disposable plastic apron, facemask, and gloves. Dispose of all waste in a clinical waste bag, and clean and disinfect all hard surfaces, floors, chairs, door handles, sanitary fittings, and reusable non-invasive care equipment with a combined detergent disinfectant solution at a dilution of $1000 \mathrm{ppm}$ available chlorine (use $10000 \mathrm{ppm}$ for equipment contaminated with blood). ${ }^{10}$ All non-disposable items used for patient care that cannot be cleaned with detergent and disinfectant should be put in a clinical waste bag. Patient care equipment should be single-use items if possible. Reusable (communal) non-invasive equipment should as far as possible be allocated to the individual patient or cohort of patients and be decontaminated with appropriate wipes between each patient.

Debrief with the practice team regularly, especially those who are directly involved in patient management. Identify vulnerable healthcare workers such as pregnant or immunosuppressed individuals and move them to non-patient facing roles and seek advice from the occupational health service if available. Staff working in reception and communal areas who are not involved in direct patient care should maintain social distancing of two meters. If this is not practical, use of a fluid resistant surgical mask is recommended. ${ }^{10}$ If a member of staff develops a fever $>37.8^{\circ} \mathrm{C}$ or respiratory symptoms, or they live in the same household as a person with symptoms, they should follow the local policy for testing and self-isolation.

Self-isolation at home for people suspected or confirmed with covid-19 infection who are not critically ill ${ }^{12}$

Anyone with symptoms of covid-19 should self-isolate to protect others in the community (resources in box 1 offer further guidance).

- Self-isolation means staying indoors for seven days from the date of onset of symptoms, avoiding contact with other people, and separating themselves from vulnerable members of the household as much as possible

- Asymptomatic household contacts should self-isolate for 14 days

- Patients who are self-isolating are advised to

- Wash hands with soap and water for 20 seconds regularly or use a hand sanitiser

- Stay at least two metres away from vulnerable individuals, including anyone over 70 , pregnant women, and anyone under 70 with underlying medical conditions that would qualify them for annual flu vaccination on health grounds

- Have food, medication, and supplies delivered to their home

- Cover coughs and sneezes with tissues and put them in a bin

- Avoid going out (except if advised to seek medical care) and not use public transport or taxis. Own vehicle may be used.

- Not have visitors at home

- Double bag and seal all waste. Keep this aside for at least 72 hours before putting it into the usual external household waste bin.

- Further information on self-isolation is available on the PHE website (see resources, box 1)

\section{Questions patients might ask about covid-19}

How do I know if I am infected with the virus?

If you have fever, new persistent cough, or feel breathless you may be infected. Please self-isolate for seven days at home and if your symptoms worsen contact NHS 111 online or by phone.

\section{Is it contagious?}

Yes, although we do not yet fully understand the precise routes of transmission. The virus is transmitted in respiratory droplets and can be spread by coughing, sneezing, or touching infected surfaces. Coronaviruses have also been detected in blood, faeces, and urine.

\section{What precautions do I need to take to prevent infection?}

Wash your hands frequently, especially after using public transport. Avoid touching your eyes and nose, and sneeze or cough into a tissue. Common disinfectants such as soaps and alcohol based hand rub are effective in eliminating the virus if it is on your hands. Face masks for the general public are not recommended.

What is the treatment for covid-19?

Most people do not need any treatment apart from fluids and paracetamol. Those who are ill will receive supportive care to help them recover from the illness in specialist settings.

\section{Education into practice}

- How aware are staff in your practice of the latest advice regarding covid-19?

- Can you describe a clear protocol for identifying and isolating patients with possible covid-19 as quickly as possible if they call for advice or present to the surgery?

- Do you have the facilities and training for remote triaging such as video consultation and a policy for its safe and effective use?

- Do you have personal protective equipment in the practice including surgical face masks, aprons, and gloves?

- Do you have the telephone number for the local infection specialist to call for advice?

- Do you have a clear process for seeking advice and referring to occupational health for any affected staff members? 


\section{How this article was made}

This article uses international websites, recent research papers, and the latest advice from Public Health England and Health Protection Scotland on identifying and managing patients with suspected covid-19 in primary care.

Competing interests The BMJ has judged that there are no disqualifying financial ties to commercial companies. The authors declare the following other interests: none.

Further details of The BMJ policy on financial interests are here: https://www.bmj. com/about-bmj/resources-authors/forms-policies-and-checklists/declarationcompeting-interests

Provenance and peer review: commissioned, based on an idea from the author; externally peer reviewed.

1 Public Health England. covid-19: epidemiology, virology and clinical features. 2020. https: //www.gov.uk/government/publications/wuhan-novel-coronavirus-background-information/ wuhan-novel-coronavirus-epidemiology-virology-and-clinical-features

2 World Health Organization. Coronavirus disease 2019 (covid-19) Situation Report - 29. 19 February 2020. Available from: https://www.who.int/docs/default-source/coronaviruse/ situation-reports/20200219-sitrep-30-covid-19.pdf?sfvrsn=6e50645_2

3 Li Q, Guan X, Wu P, etal . Early transmission dynamics in Wuhan, China, of nove coronavirus-infected pneumonia. N Engl J Med 2020.

10.1056/NEJMoa2001316 31995857

4 Guan W, Ni Z, Hu Y, etal . Clinical Characteristics of Coronavirus Disease 2019 in China. N Engl J Med 202010.1056/NEJMoa2002032.
5 World Health Organization. WHO Director-General's remarks at the media briefing on COVID-2019 outbreak on 17 February 2020. $2020 \mathrm{https} / /$ www.who.int/dg/speeches/detail/ who-director-general-s-remarks-at-the-media-briefing-on-covid-2019-outbreak-on-17february-2020.

6 Wu Z, McGoogan JM. Characteristics of and important lessons from the coronavirus disease 2019 (covid-19) outbreak in China. Summary of a report of 72314 cases From the Chinese Center for Disease Control and Prevention. JAMA 2020.

10.1001/jama.2020.2648 32091533

7 England Guidance NHS. Managing coronavirus (covid-19) in general practice (SOP) 2020. https://www.england.nhs.uk/coronavirus/publication/managing-coronavirus-covid19-in-general-practice-sop_19-march/

8 Public Health England Guidance. covid-19: investigation and initial clinical management of possible cases. 2020. https://www.gov.uk/government/publications/wuhan-novelcoronavirus-initial-investigation-of-possible-cases/investigation-and-initial-clinicalmanagement-of-possible-cases-of-wuhan-novel-coronavirus-wn-cov-infection\#interimdefinition-possible-cases

9 Public Health England. Guidance on shielding and protecting people defined on medical grounds as extremely vulnerable from COVID-19. 2020. Available from: https://www.gov. uk/government/publications/guidance-on-shielding-and-protecting-extremely-vulnerablepersons-from-covid-19/guidance-on-shielding-and-protecting-extremely-vulnerable-personsfrom-covid-19

10 Public Health England. COVID-19: infection prevention and control guidance. 2020. https: //www.gov.uk/government/publications/wuhan-novel-coronavirus-infection-preventionand-control

11 Public Health England. covid-19: interim guidance for primary care. Available from: https: //www.gov.uk/government/publications/wn-cov-guidance-for-primary-care/wn-cov-interimguidance-for-primary-care

12 Public Health England. Stay at home: guidance for households with possible coronavirus (COVID-19) infection. 2020. Available from: https://www.gov.uk/government/publications/ covid-19-stay-at-home-guidance/stay-at-home-guidance-for-households-with-possiblecoronavirus-covid-19-infection

Published by the BMJ Publishing Group Limited. For permission to use (where not already granted under a licence) please go to http://group.bmj.com/group/rights-licensing/ permissions 


\section{Figure}
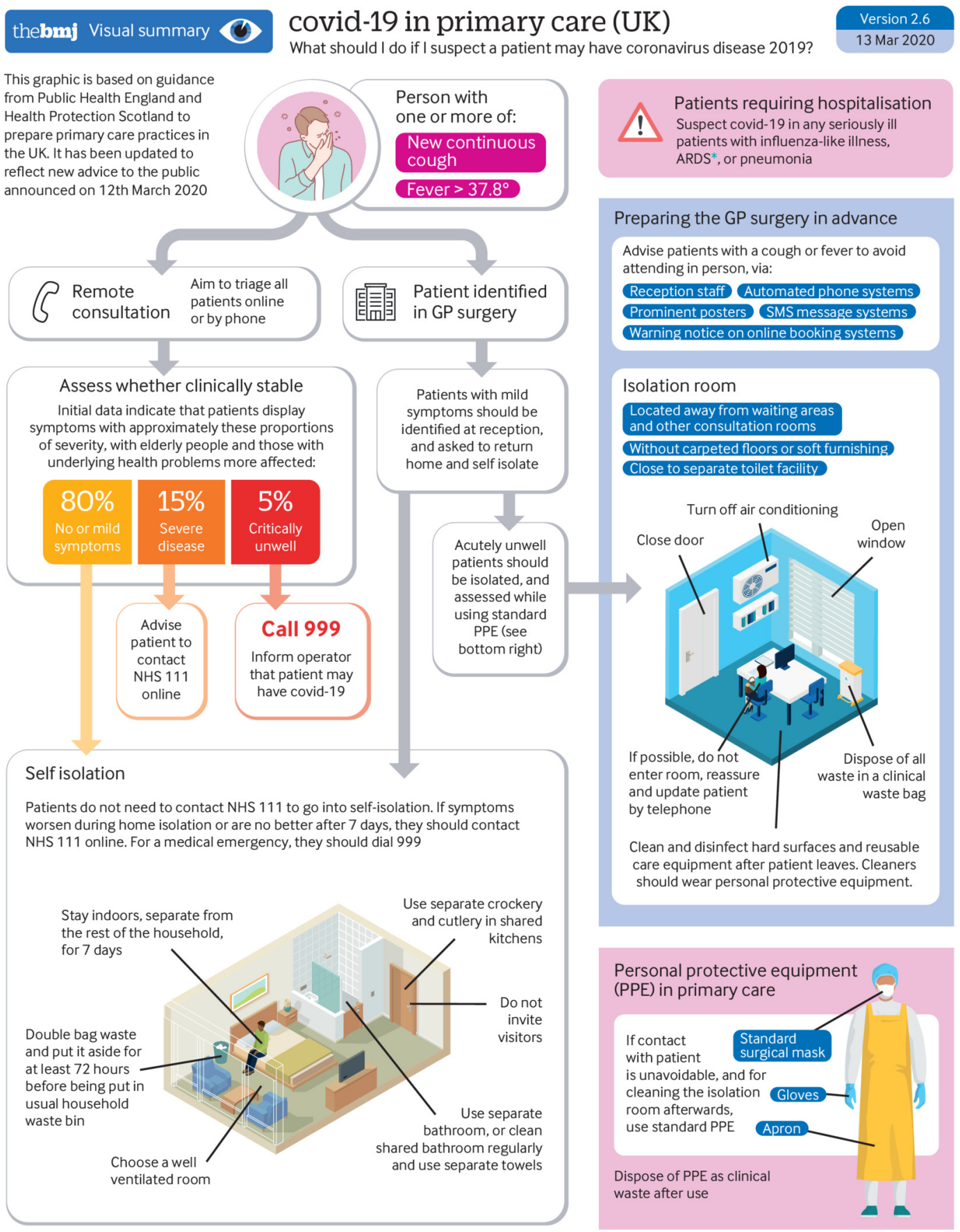

* ARDS = acute respiratory distress syndrome

(c) 2020 BMJ Publishing Group Ltd.

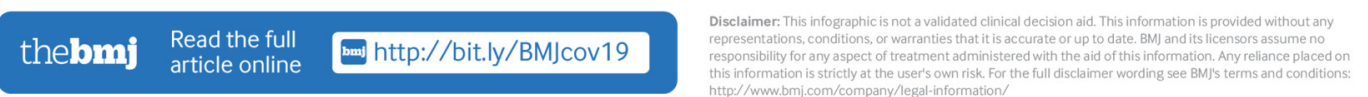

